University of Nebraska - Lincoln

DigitalCommons@University of Nebraska - Lincoln

2010

\title{
Multimodal courtship efficacy of Schizocosa retrorsa wolf spiders: Implications of an additional signal modality
}

\author{
Aaron S. Rundus \\ University of Nebraska-Lincoln, arundus2@unl.edu \\ Roger D. Santer \\ University of Limerick, Ireland, roger.santer@ul.ie \\ Eileen Hebets \\ University of Nebraska - Lincoln, ehebets2@unl.edu
}

Follow this and additional works at: https://digitalcommons.unl.edu/bioscihebets

Part of the Behavior and Ethology Commons

Rundus, Aaron S.; Santer, Roger D.; and Hebets, Eileen, "Multimodal courtship efficacy of Schizocosa retrorsa wolf spiders: Implications of an additional signal modality" (2010). Eileen Hebets Publications. 43.

https://digitalcommons.unl.edu/bioscihebets/43

This Article is brought to you for free and open access by the Papers in the Biological Sciences at DigitalCommons@University of Nebraska - Lincoln. It has been accepted for inclusion in Eileen Hebets Publications by an authorized administrator of DigitalCommons@University of Nebraska - Lincoln. 
Published in Behavioral Ecology 21:4 (2010), pp. 701-707; doi: 10.1093/beheco/arq042 Copyright (C 2010 Aaron S. Rundus, Roger D. Santer, and Eileen A. Hebets; published by Oxford University Press on behalf of the International Society for Behavioral Ecology. Used by permission.

Submitted September 6, 2009; revised February 5, 2010; accepted February 10, 2010; published online April 13, 2010.

\title{
Multimodal courtship efficacy of Schizocosa retrorsa wolf spiders: Implications of an additional signal modality
}

\author{
Aaron S. Rundus, ${ }^{1}$ Roger D. Santer, ${ }^{2}$ and Eileen A. Hebets ${ }^{1}$ \\ 1. School of Biological Sciences, University of Nebraska-Lincoln, 325 Manter Hall, Lincoln, NE 68588, USA \\ 2. Department of Life Sciences, Schrödinger building, University of Limerick, Limerick, Ireland \\ Corresponding author - A. S. Rundus, email arundus2@unl.edu
}

\begin{abstract}
Here, we simultaneously examine both content and efficacy-based sources of selection on the visual and seismic multimodal courtship display of the wolf spider Schizocosa retrorsa. Immature field-collected S. retrorsa were reared in the laboratory on either high-quantity diet (HD) or low-quantity diet (LD) treatments. On maturation, females of each diet treatment were run in simultaneous mate choice trials with both a HD and an LD male (content-based selection). Simultaneous mate choice trials were conducted across different signaling environments (efficacy-based selection) in a fully crossed $2 \times 2$ design with visual treatments of light/dark (visual signal present/absent) and seismic treatments of filter paper substratum/granite substratum (seismic signal present/absent). Male copulation success did not differ across either signaling environment or diet treatment. However, copulation success was related to male leg waving displays as males who engaged in more bouts of leg waving were more likely to copulate regardless of experimental manipulations. The observation that copulation success was independent of signaling environment suggests the use of an additional courtship signal modality.
\end{abstract}

Keywords: courtship performance, mate choice, sexual selection, signal content, signal efficacy

Within the animal kingdom, the form and deployment of communicative signals is astonishingly diverse. Historically, efforts to explain the variation found in animal signals have largely focused on single signals, often in isolation. More recently, however, investigations of signal evolution and function have begun to recognize the importance of complex signals (for reviews see; Rowe 1999; Partan and Marler 1999, 2005; Candolin 2003; Hebets and Papaj 2005). Complex signals are those that are composed of multiple components that utilize either the same or different modalities, the latter being referred to as a multimodal signal (Guilford and Dawkins 1991; Partan and Marler 1999; Rowe and Guilford 1999). The prevalence of complex signaling across a wide variety of taxa suggests that in many contexts, complex signals may reflect a selective advantage to signaling participants. For example, signalers may benefit from using a complex signal in highly variable environments (e.g., Candolin 2003; Hebets and Papaj 2005) or when signal targets are capable of perceiving a signal through multiple sensory channels (e.g., Rundus et al. 2007). Likewise the targets of complex signals may receive benefits through an increased potential for signaler assessment (Møller and Pomiankowski 1993; Johnstone 1996).

A richer understanding of the evolution of complex animal signals necessitates an examination of the sources of selection that may have shaped their form and deployment. These sources of selection can be divided into 2 major categories: content-based selection and efficacy-based selection (Guilford and Dawkins 1991; Hebets and Papaj 2005). Content-based selection, or strategic design, relates to how a signal is designed to encode the information necessary for assessment by a perceiving animal (e.g., "multiple messages" and "redundant" or "backup signals," Møller and Pomiankowski 1993; Johnstone 1996). Another way of thinking about strategic design is in how the design characteristics of a signal capital- ize on or reflect perceiver concerns (e.g., signaler size, condition, and motivational state). Efficacy-based selection, in contrast, relates to how a signal is designed to propagate through the environment and elicit a response in the signal target $(\mathrm{Ow}-$ ings and Morton 1998; for empirical example see Fleishman 1986). Because both sources of selection are likely to act simultaneously, thorough investigations of complex signal function would benefit from an experimental design that examines both content- and efficacy-based sources of selection, yet most studies to date utilize only one approach (Partan and Marler 1999; Candolin 2003; Hebets and Papaj 2005). Here, we attempt to simultaneously examine both of these sources of selection in the multimodal courtship display of the wolf spider Schizocosa retrorsa.

Schizocosa wolf spiders are an ideal model system for studying multimodal signals. Members of this genus vary considerably with regard to both male secondary sexual traits and courtship displays (for reviews see Stratton 2005; Framenau and Hebets 2007). Male Schizocosa courtship always includes seismic components (substratum-coupled stridulation, tremulation, or drumming) with some species also incorporating visual components (leg waving, leg arching, and body bouncing) (Stratton 2005). The visual components have been shown to be condition-dependent in S. ocreata and S. uetzi (Uetz et al. 2002; Hebets, Wesson, and Shamble 2008; Shamble et al. 2009), suggesting that they are at least partially under content-based selection. Historically, investigations of Schizocosa courtship have examined the efficacy of displays by assessing female responses to signal components in isolation. Signal isolation has been achieved either by ablating/masking one of the signal components (Hebets and Uetz 1999; Uetz and Roberts 2002; Gibson and Uetz 2008) or in the case of visual signals, through the use of video playbacks of artificial spider stimuli (Hebets and Uetz 2000; Uetz and Roberts 2002; Hebets 2005, 2008; Uetz 
and Norton 2007). Under these scenarios, signal efficacy has been explored by measuring both female receptivity to isolated signal components (e.g., Scheffer et al. 1996; Hebets and Uetz 1999; Gibson and Uetz 2008) and by examining mating success under environmental conditions enabling signal isolation (Hebets 2005, 2008; Hebets et al. 2006). In these studies, the successful elicitation of female receptivity and/or successful copulation demonstrates an efficacious signal. In such scenarios, however, it is important to note that a lack of a female response does not necessarily imply an ineffective signal but instead may simply reflect a female's decision not to respond.

Here, we simultaneously use both content- and efficacybased approaches to examine the multimodal (visual and seismic) courtship display of the ornamented Schizocosa wolf spider S. retrorsa (banks). This species is typically found in highly exposed areas on pine needles, red clay, sandy substrates, or some combination of the 3 (Hebets et al. 1996). Mature males possess black pigmentation on the femora of their forelegs and produce courtship displays traditionally thought of as a series of visual and seismic components (Hebets et al. 1996). Male courtship often begins with a push-up behavior, where a male raises his body up onto the tips of his legs producing a vibration audible to human observers as a "click," assumed to be generated via stridulation. Courtship progresses with the occurrence of pedipalpal drumming in which the male alternately lifts and lowers his 2 pedipalps to the substrate-producing a seismic signal. Pedipalpal drumming often accompanies a third component, extended leg waves (previously called taps in Hebets et al. 1996), where one or both ornamented forelegs are extended, lifted, lowered, and then tapped on the substrate extremely rapidly. All 3 of these courtship behaviors include both visual and seismic components. By manipulating the signaling environment, as well as the foraging history (and putatively body condition) of male and female $S$. retrorsa and assessing copulation success as a first proxy of signal efficacy (e.g., successful copulation indicates an efficacious signal), we aimed to determine the relative importance of content- and efficacy-based selection on the function of male $S$. retrorsa courtship displays.

\section{Materials and Methods}

\section{Subjects}

A total of 238 (100 male and 138 female) wild-caught $S$. retrorsa were run through simultaneous mate choice trials. All spiders were collected from the field as subadults from Lafeyette County, MS, USA on 23 May 2007 ensuring that all individuals were virgins. Spiders were brought back to the laboratory where they were individually housed in $5.9 \times 5.9$ $\times 7.7 \mathrm{~cm}$ clear plastic containers (Amac Plastic Products, Petaluma, CA) with visual barriers between containers. Spiders were maintained on a 12:12 light:dark cycle and provided with a constant source of water. In order to manipulate body condition in both males and females, all individuals were randomly assigned to one of 2 diet treatments: 1) low-quantity diet (LD): one cricket (Acheta domesticus) (Bassetts cricket ranch, CA) of a size that visually approximated the body length of the spider (prosoma + opisthosoma) once every 2 weeks and 2) highquantity diet (HD): one cricket size-matched as above twice per week. All spiders were examined every 2 days for molts in order to accurately estimate the date of their final maturation molt. In females, we manipulated diet in order to examine whether female choice varies with foraging history because female diet has been previously shown to influence mate choice in another Schizocosa wolf spider (Hebets, Wesson, and Shamble 2008). In males, we manipulated diet in order to generate variation in male body condition to examine if and how male body condition influences female mate choice.

\section{Experimental design}

Simultaneous mate choice trials utilized a fully crossed $2 \times$ 2 experimental design with a visual treatment of light versus dark (visual signal present/absent) and a seismic treatment of filter paper substratum versus granite substratum (seismic signal present/absent). The experimental protocol employed in this study was similar to Hebets (2005). Briefly, visual signal present trials were run under 2 Vita-Lite full spectrum 30-W florescent bulbs (Duro-Test Lighting Inc., Philadelphia, PA), whereas visual signal absent trials were run under illumination solely from a Supercircuits IR20 infrared illuminator (Supercircuits, Austin, TX) in the absence of visible spectrum light (0 Lux measured using a VWR Scientific dual range light meter model 62344-944, West Chester, PA). These trials were observed using Rigel 3200 night vision goggles (Rigel Optics Inc., Washougal, WA). The IR illuminator emitted wavelengths of $\sim 850 \mathrm{~nm}$, which has been suggested as an acceptably high "undetectable" wavelength for studies of crustaceans, which may have some sensitivity to near infrared light (Lindström and Meyer-Rochow 1987). The principle eyes of arachnids are of the camera type, unlike those of insects and crustaceans, and currently little is known of their spectral sensitivities. Available data on various wolf spiders, and on the wandering spider Cupeinnius salei, provide no indication that they can detect IR wavelengths in excess of $800 \mathrm{~nm}$ (DeVoe et al. 1969; DeVoe 1972; Barth 2002). Observations from all visual-signal absent trials were voice recorded on a digital recorder for later transcription and analysis. Seismicabsent trials were run on a piece of granite that, like other types of rock, does not transmit seismic signals effectively (Elias et al. 2004). Prior recordings of seismic signals produced by a larger drumming species of Schizocosa, S. mccooki, were made using a laser Doppler vibrometer (Polytec PDV100) on our granite substrate, and we were unable to visualize an obvious seismic signal (Rundus AS, unpublished data), further supporting the notion that granite successfully impedes seismic signal transmission. Seismic-present trials were run on a piece of Whatman \#1 185-mm filter paper (Schleicher and Schuell, Keene, NH). On the granite, spiders were enclosed within a bottomless circular plastic arena measuring 20.5-cm diameter (Amac Plastic Products, Petaluma, CA). Spiders in the seismic-present treatments were enclosed in the same sized plastic arenas lined with filter paper.

\section{Mating trials}

To control for time of day effects, a group of 4 visualpresent trials were always run back-to-back with a group of 4 visual-absent trials. Visual-present trials were comprised 2 replicates of the treatments seismic-present $(\mathrm{V}+/ \mathrm{S}+)$ and seismic-absent $(\mathrm{V}+/ \mathrm{S}-)$. Similarly, visual-absent trials consisted of 2 replicates of seismic-present $(\mathrm{V}-/ \mathrm{S}+)$ and seismic-absent (V-/S-). Approximately $24 \mathrm{~h}$ prior to testing, all females (regardless of their prior diet treatment) were provided a single cricket, size-matched to one half of the female's body size in order to increase the likelihood that they would be reproductively motivated as opposed to nutritionally motivated. A minimum of $48 \mathrm{~h}$ prior to experimental trials, HD and LD males were marked with either a black or a white dot of paint (Deco color paint pens; Uchida of America, Corp., Torrance, CA) on their cephalothorax in order to allow individual identification. Immediately prior to the start of the trial, males and females were weighed (Ohaus Adventurer Pro AV64, Pine Brook, NJ), and the females were subsequently placed in their arena and allowed to acclimate for $5 \mathrm{~min}$. After this acclima- 
tion period, the HD and LD male spiders were placed in the testing arena, on opposite sides under inverted glass vials. Males in each arena were age-matched to within 4 days postmaturation. Trials commenced with the simultaneous removal of the inverted vials and all 3 individuals in each arena were allowed to interact for $45 \mathrm{~min}$. Trials were scored for the following: all occurrences of male courtship, copulation, cannibalism, and male attempted mounts. We also conducted instantaneous sampling every 5 min for each male's behavior, and we recorded the times to first courtship and copulation for every male. All conventional statistical tests were carried out using JMP v7.01 (SAS Institute Inc., Cary, NC); we additionally carried out Mantel randomization tests using Mantel. XLA-VBA v 1.2.4 (Briers 2003).

\section{Results}

A total of 138 females (LD: $N=69$; HD: $N=69$ ) and 100 males (LD: $N=50$; HD: $N=50$ ) were run through mate choice trials. Female spiders were maintained on their assigned diet treatment for an average of 40.4 days and males for an average of 42.3 days. To assess the effectiveness of our diet manipulations, we calculated a body condition index (mass [g]/cephalothorax width $[\mathrm{cm}]$ ) for both male and female spiders. Cephalothorax measures were taken on spiders (preserved in 70\% ethanol) at the conclusion of the experiment. Body condition scores could not be calculated for 24 females (9 LD and 15 HD) and 13 males (6 LD and $7 \mathrm{HD}$ ) due to decomposition or postcopulatory cannibalism. At the time of testing, females on the HD treatment had a significantly higher body condition score than those on the LD treatment (HD: mean \pm standard error $[\mathrm{SE}]=0.042 \pm 0.0007 ; \mathrm{LD}$ : mean $\pm \mathrm{SE}=0.025 \pm 0.0007$; $t$-test: $\left.t_{112}=17.029, P<0.0001\right)$. As with females, on the day of their trials, HD males had higher body condition scores than their paired LD male (HD: mean $\pm \mathrm{SE}=0.017 \pm 0.0002$; LD: mean $\pm \mathrm{SE}=0.015 \pm 0.0002$; paired samples $t$-test: $t_{124}=3.812, P=$ $0.0002)$. Female age at the time of testing ranged from 18 to 32 days postmaturity with a mean age of 23.02 days. HD females were significantly older than LD females by just over one day (LD: mean $\pm \mathrm{SE}=22.2 \pm 0.22$ days; $\mathrm{HD}$ : mean $\pm \mathrm{SE}=23.9 \pm 0.36$ days; Wilcoxon test: $Z=3.65$, degrees of freedom [df] $=1, P$ $<0.001)$. This difference in female age was primarily due to differences in maturation time between the 2 diet treatments. Because LD individuals took longer to mature, we needed to wait until LD males were mature and thus available before running HD females - necessarily making HD females slightly older at the time of testing than LD females. Within a trial, LD males averaged 33.5 days postmaturation, whereas HD males averaged 32.9 days postmaturation. A paired $t$-test using individuals for which we have accurate age data $(N=129)$ reveals that this difference in age is significant $\left(t_{128}=2.12, P=0.04\right)$.

Due to a limited number of males given our 2-choice design, males were used in multiple trials -26 males were used twice (12 HD; 14 LD), 33 males were used 3 times (14 HD; 19 LD), and 28 males were used in 4 trials (16 HD; 22 LD). Each individual female spider was used in only one trial. Although males were used multiple times, each individual male-male LD-HD pairing was unique, and males were only run once in any given treatment condition. Multiply used males had a minimum of 3 days in between trials with an average of 9.6 days. There was no significant difference in the distribution of males that were used in 1, 2, 3, or 4 trials across the 4 environmental treatment conditions $\left(\chi^{2}=7.7, P=0.57\right)$. To determine whether mating success in a male's initial trial would affect the mating success of that male in subsequent trials, we examined the mating patterns of multiply used males across their initial and subsequent mate choice trial. We found no dif- ference in a male's likelihood to copulate based on his initial experience $\left(\chi^{2}=2.1, P=0.56\right)$, and males who either mated in every trial (i.e., studs) or in none of their trials (i.e., duds) were equally likely to be HD or LD males $\left(\chi^{2}=0.005, P=0.95\right)$. Furthermore, using a nominal logistic regression, we found that $\mathrm{HD}$ and LD male trial number did not predict the occurrence of copulation in a trial $\left(\chi^{2}=1.66, \mathrm{df}=2, P=0.436\right)$.

We examined the effect of signaling environment on copulation success using a Mantel test (Sokal and Rohlf 1995). This nonparametric randomization test estimates the association between 2 dissimilarity matrices representing the same set of entities (in our case trials) and tests whether this association is greater than would be expected by chance. The Mantel test is particularly well suited to comparing outcomes from multiple trials involving a limited and repeated set of subjects without assuming independence of observations (see e.g., Draud et al. 2004). In our case, we tested the association between one matrix representing dissimilarity in environmental testing condition between trials and a second matrix representing dissimilarity in the occurrence of copulation between trials. We performed the Mantel test using based on 20,000 randomizations. We found no significant correlation between the 2 matrices (Mantel $Z: R$ $=-0.01, P=0.172$; Figure $1 \mathrm{~A})$, indicating that dissimilarities in copulation occurrence did not correlate with dissimilarities in environmental treatment or in other words that variation in copulation occurrence was not greater between the conditions than it was within them (Sokal and Rohlf 1995).

Even though experience did not appear to influence subsequent mating success, we did not conduct further, more conventional, statistical analyses on our complete data set in order to avoid violations of independence. Thus, all following analyses include only the first trial for every male-removing any statistical problems associated with pseudoreplication. Although this greatly reduces our sample size ( $N=49$ vs. $N$ $=138$ ), the observed pattern of copulation success and mate choice in this reduced data set is similar to that observed using the complete data set (Figure 1A vs. Figure 1B).

In order to determine if signaling environment and/or female diet influenced copulation success, we ran a nominal logistic model with female diet and signaling environment as the main factors and female age as a random factor. Although female age only varied by a day between HD and LD females, age has been shown to influence female receptivity in $S$. ocreata (Uetz and Norton 2007), and thus, we included it in our analyses. We found no influence of female diet, signaling environment, or female age on the likelihood of copulation (whole model test: $\left.\chi^{2}=10.6, \mathrm{df}=8, P=0.225\right)$ or on which male (HD vs. LD) achieved the copulation (whole model test: $N=25, \chi^{2}$ $=8.83, \mathrm{df}=8, P=0.356$; Figure $1 \mathrm{~B}$ ).

Within the 49 trials representing each male's first use, at least one male courted in $84 \%$ of trials and both males courted in $35 \%$. HD males were the first to court in $62 \%$ of trials and this does not differ from the null expectation of 50:50 $\left(x^{2}=2.1\right.$, $P=0.15)$, indicating that HD and LD males are equally likely to be the first to court. On average, across all trials, HD males engaged in significantly more bouts of leg waving than LD males (LD: mean $\pm \mathrm{SE}=1.51 \pm 0.51$ bouts; HD: mean $\pm \mathrm{SE}=$ $6.49 \pm 1.65$ bouts; paired samples $t$-test: $t_{48}=2.81, P=0.0071$; Figure 2A). Additionally, HD males engaged in more attempted mounts on average than LD males (LD: mean $\pm \mathrm{SE}=$ $0.12 \pm 0.05$; HD: mean $\pm \mathrm{SE}=0.79 \pm 0.23$; paired samples $t$-test: $t_{48}=2.94, P=0.0049$; Figure 2B).

In analyzing only the pairs for which a copulation occurred, there was no significant difference in courtship effort between HD and LD pairs, as measured by the number of leg waving bouts prior to copulation (LD: mean \pm SE $=1.4 \pm 0.46$; $\mathrm{HD}$ : mean $\pm \mathrm{SE}=3.04 \pm 0.95$; paired samples $t$-test: $t_{24}=1.71$, $P=0.1$ ). There was also no difference in the time between first 

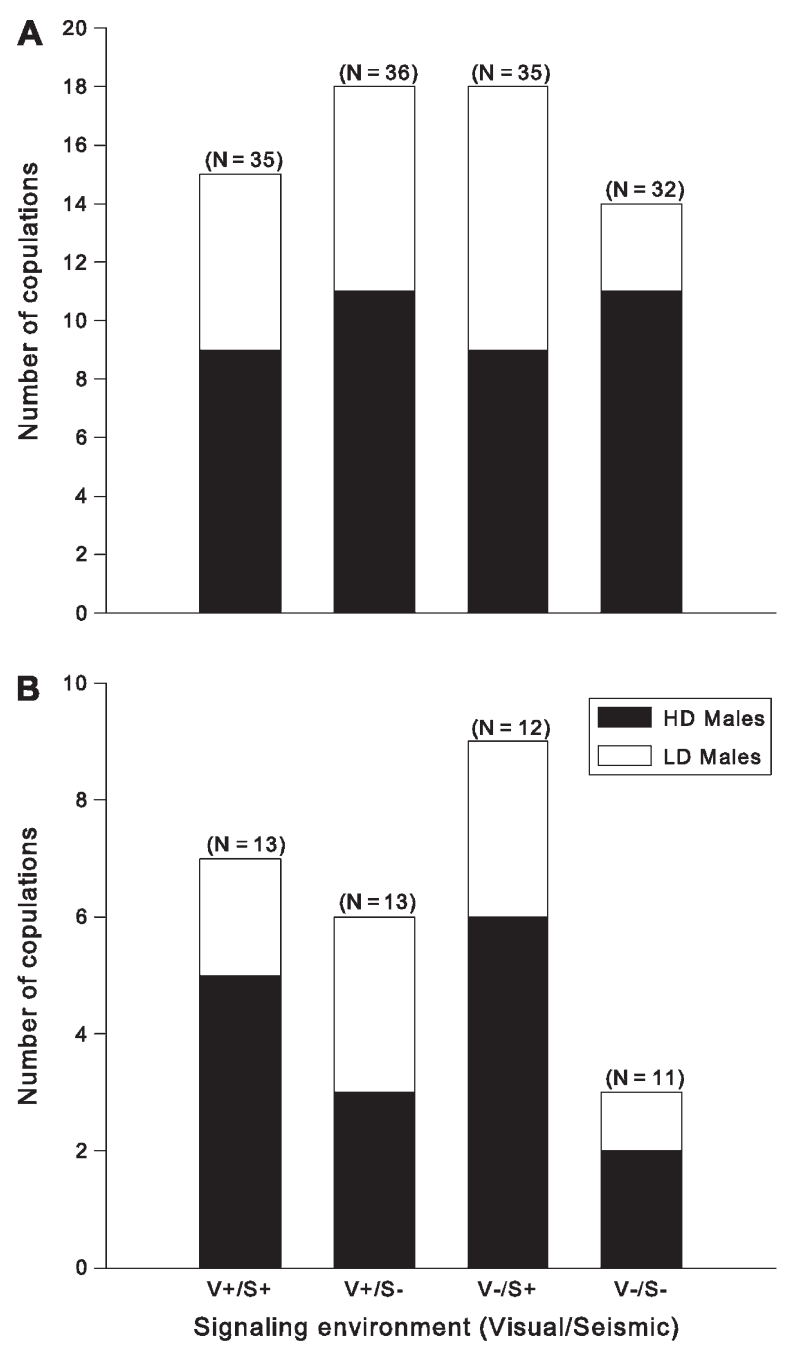

Figure 1. The number of trials resulting in copulation by HD and LD males across the 4 environmental testing conditions. Panel (A) represents data from all trials $(N=138)$ including repeated trials with the same males, and panel (B) represents data from only the first trials for male spiders $(N=49)$. As with the full data set, our reduced data set showed no difference in copulation success across the 4 environmental testing conditions $\left(\chi^{2}=5.408, \mathrm{df}=3, N=49, P=0.144\right)$

courtship and copulation for HD versus LD copulating males $(\mathrm{LD}$ : mean $\pm \mathrm{SE}=238.6 \pm 121.5 \mathrm{~s} ; \mathrm{HD}$ : mean $\pm \mathrm{SE}=262.4 \pm 80.5$ s; Wilcoxon test: $Z=0.02, \mathrm{df}=1, P=0.88$ ). However, it was the male who engaged in more courtship bouts that was more likely to achieve copulation (Wilcoxon test: $x^{2}=8.58, \mathrm{df}=1, P$ = 0.003; Figure 3).

\section{Discussion}

Despite the seemingly conspicuous nature of both the visual and seismic courtship signals of $S$. retrorsa, our manipulations of the signaling environment suggest that neither signal is necessary for successful copulation. Pairs were equally likely to copulate across all signaling environments, indicating that the courtship display of males is effective regardless of the successful transmission of visual and/or seismic display components. Curiously, irrespective of signaling environment, a male's number of leg waving bouts was a good predictor of copulation success - suggesting effective courtship even in the absence of visual and/or seismic components. The observed ef-
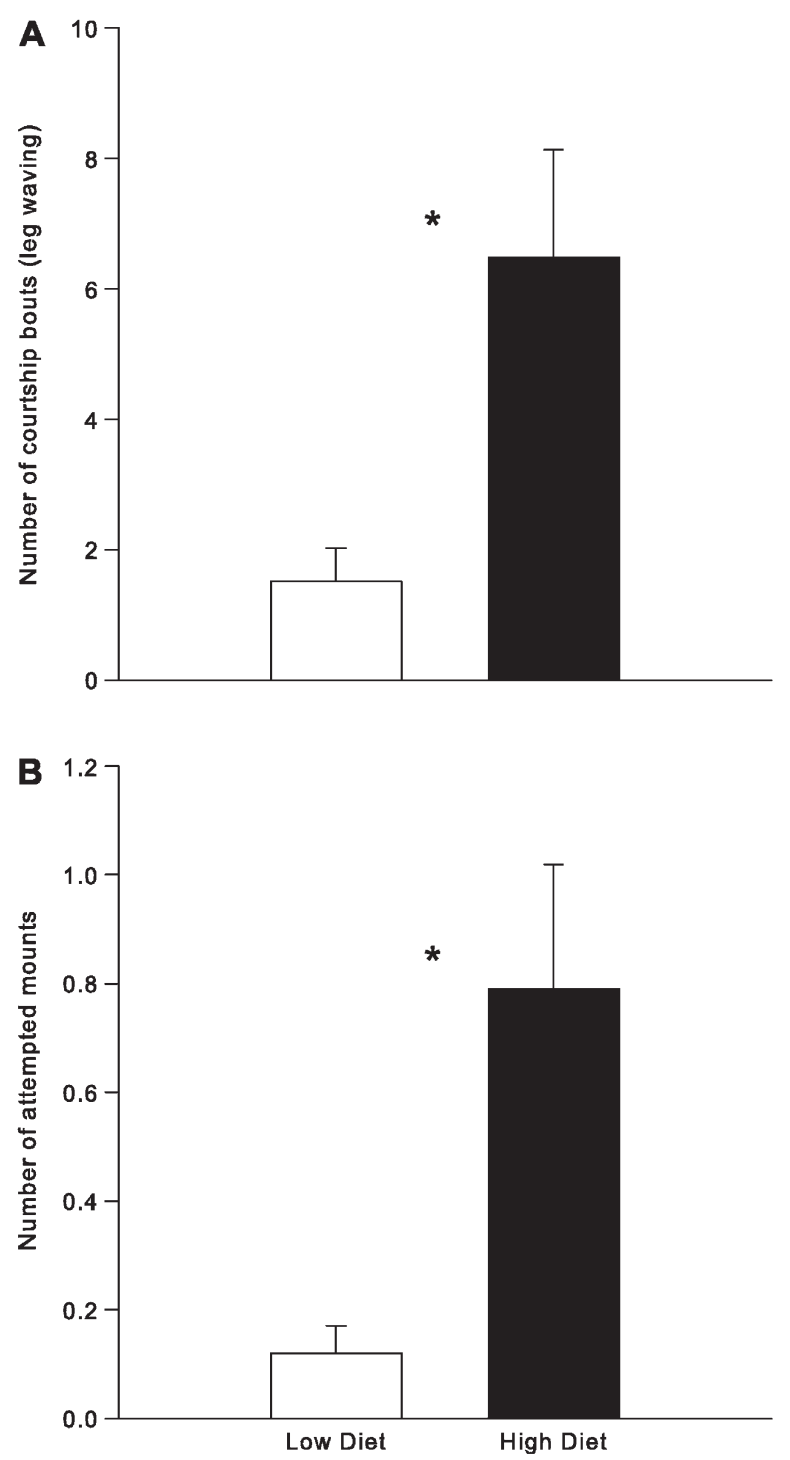

Figure 2. The mean number of (A) bouts of courtship and (B) attempted mounts by HD and LD males prior to a copulation taking place within a trial. Error bars represent SE's of the means. ${ }^{*} P<0.01$.

ficacy of male S. retrorsa courtship even in the V-/S- signaling treatment suggested to us the use of an additional signaling modality, which we will discuss below. Finally, mating frequencies were independent of individual diet treatments both within and across signaling environments, demonstrating that our diet manipulations did not influence the mating behavior or mate choice of $S$. retrorsa, and potentially suggesting that efficacy-based selection may be more important in this species.

Our diet manipulations generated significant differences between the HD and LD treatment individuals with regard to their body condition score, but copulation success did not depend on either the male or the female diet treatment. Despite this lack of dependence, the possibility exists that individuals were not on their diet treatments for long enough during development to observe differences in mate choice. The immature spiders we collected from the field were of unknown age-making it impossible to know a priori how far away they were from sexual maturity. Despite this limitation, our data provide no support for the notion that females are choosing males based on body condition in this species. 


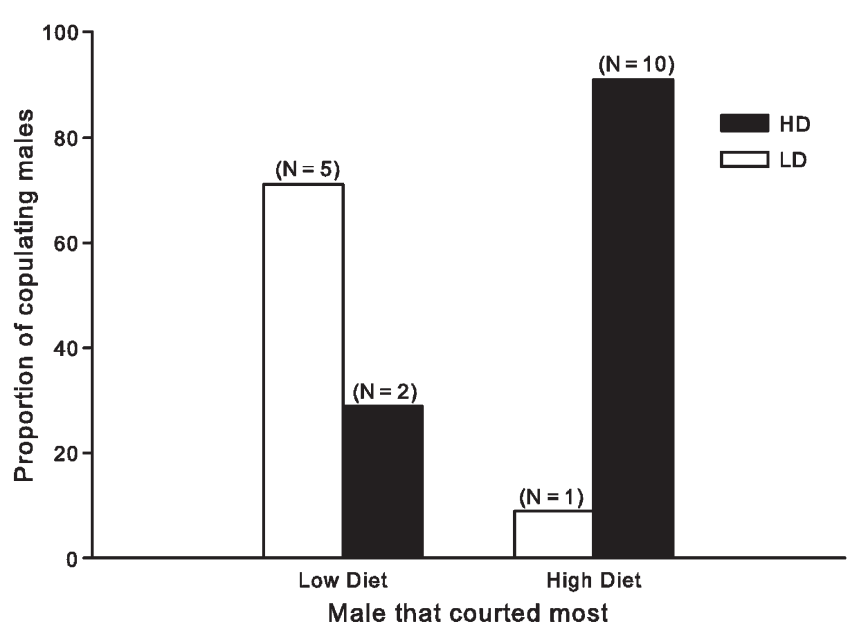

Figure 3. The proportion of copulating males that were HD or LD during trials where either an LD male or a HD male courted the most. Data shown are from trials in which a copulation occurred.

Our manipulations of the signaling environment enabled us to examine the necessity of both visual and seismic courtship components. We found no significant difference in male copulation success when the visual and seismic components were either both present, presented in isolation, or both ablated. The pattern of copulation success independent of the signaling environment is clearly evident in the full data set in which males were used multiple times (Figure 1A). In the reduced data set (Figure 1B), there does appear to be a slight decrease in copulation success when both signaling modalities are removed $\left(\mathrm{V}-/ \mathrm{S}^{-}\right)$, although this decrease is not significant. Regardless, even in this reduced data set, close to $30 \%$ of females still copulated in the absence of visual and seismic courtship signals, and this pattern is identical to that previously reported for $S$. retrorsa (see Figure 2 in Hebets and Papaj 2005). Thus, even using the reduced data set, S. retrorsa pairs appear capable of successfully copulation in the dark, on granite-presumably in the absence of both visual and seismic courtship signals.

One possible explanation for effective male courtship in the absence of visual and seismic signal transmission is that we did not successfully ablate our signals. However, we are relatively confident that this is not the case for the following reasons. First, our visual-absent treatments were run solely under infrared illumination at $\sim 850 \mathrm{~nm}$ and viewed through infrared goggles. Although the visual sensitivity of $S$. retrorsa has not been directly examined, and data on arachnid eyes are rather sparse generally, in those spiders studied within the same family as $S$. retrorsa there is no evidence to suggest that their eyes can detect infrared illumination at the wavelengths emitted by our illuminator (DeVoe et al. 1969; DeVoe 1972). Until data on the visual sensitivity of $S$. retrorsa are available, we cannot completely rule out the possibility of a visual component in the visual-absent signaling environment, but at present it seems unlikely. Second, we were unable to detect an obvious seismic signal from prior recordings taken from another larger drumming Schizocosa species, S. mccooki, on granite (Rundus AS, unpublished data). Again, data directly from $S$. retrorsa are not available, but the seismic component of $S$. mccooki courtship is a good proxy for the successful transmission of drumming courtship. Furthermore, previous studies demonstrating the importance of the seismic signal for $S$. uetzi and $S$. stridulans used the same environmental manipulations as were used here (Hebets 2005, 2008; Hebets et al. 2006). Ul- timately, we believe that we were successful in reducing and/ or eliminating both visual and seismic courtship components, leading us to the conclusion that male $S$. retrorsa courtship is effective even without its seemingly conspicuous visual and seismic components.

Successful copulation does not necessarily imply accepting females. An alternative explanation to successful courtship in the $\mathrm{V}-/ \mathrm{S}-$ environment is that a male's motivation to mate and overall courtship effort supersedes female preferences for components of the male's display. For example, given the fairly confined space in which males and the female were allowed to interact, highly motivated males might have been able to capitalize on opportunities to mount females on running into them and force copulations. This seems unlikely for several reasons. First, given the high level of sexual size dimorphism found in this species (male $=5.74-7.4 \mathrm{~mm}$, female = 7.75-12.8 mm; Dondale and Redner 1978), females can almost certainly dislodge a male attempting a mount if they so choose. This is supported by a large number of observations of females dislodging males after a successful male mount (Hebets EA, Rundus AS, personal observation). Furthermore, we found no difference in the number of attempted mounts by males with regard to whether a copulation did or did not occur in the trial (Wilcoxon test: $Z=1.08, \mathrm{df}=1, P=0.28$ ).

In our view, the most likely explanation for the maintenance of male copulation success in the absence of visual and seismic courtship signals is that the complex courtship displays of male S. retrorsa can be perceived by females through one or more additional sensory modalities. This is suggested by the fact that across all environmental testing conditions, including trials run on granite in the dark, the male engaging in more courtship bouts was significantly more likely to achieve copulation in a given trial. Although courtship may also be perceived by the other male in the arena, functioning as a male-male competitive signal, it seems more likely that the signal is female directed. The presence of either a female or female silk deposited on the substrate is the primary elicitor of courtship in S. retrorsa, and courtship is most often performed when the male is oriented toward a female and not toward another male (A. S. Rundus, personal observation). The exact modality in which these courtship bouts may be sensed by females is open to debate, but we suggest 2 possibilities: male pheromones and near-field particle movements.

Male silk from the wolf spider $S$. ocreata is reported to contain pheromones that inhibit the courtship of other males (Ayyagari and Tietjen 1986) and so could potentially be a channel of chemical communication with females. Male silk cues would, however, not explain the dependence of copulation success on the number and timing of leg waves within the male courtship display. Importantly, tactile cues, either with silk or directly between the male and female, can be excluded for the same reason. Available evidence does suggests that female wolf spiders of many species, including several Schizocosa, produce sex pheromones of cuticular origin and in some cases operating via airborne transmission. These female-produced pheromones are attractive to males and elicit courtship or searching behaviors (for a review, Gaskett 2007). Although pheromones produced by males are less well known, behavioral evidence suggests that male desert spiders, Agelenopsis aperta, produce a low volatility short-range pheromone that they direct toward females through the drumming of their pedipalps (Becker et al. 2005). Similarly, the leg waves of male $S$. retrorsa could potentially serve to waft a plume of any such pheromone toward the receiving female and in that way explain female detection of the male leg wave displays even in the dark. Clearly, this possibility relies on the discovery of potential pheromones in male $S$. retrorsa, and so until this research is undertaken, this proposal must remain somewhat speculative. 
A second possibility is that male $S$. retrorsa courtship is perceived as airborne sound-pressure waves and/or particle displacements. The courtship of many male spiders, including male $S$. retrorsa, includes audible sounds made by stridulation and drumming. Males and females both have slit sensilla that could receive the pressure wave component of these sounds if sufficiently loud (Barth 1982). However, for small animals like spiders, the displacement of air particles, rather than the pressure waves they cause, is likely to be of greatest importance (Bennet-Clark 1971), and the leg wave of S. retrorsa males during courtship may produce significant air particle displacements. It is already known that many arachnids, again including $S$. retrorsa, possess extremely fine filiform hair sensilla called trichobothria. These trichobothria, and similar hairs in insects and crustaceans, are exquisitely sensitive to microscale particle displacements (e.g., Görner and Andrews 1969; Shimozawa et al. 2003). In spiders, trichobothria play a crucial role in the detection and capture of prey (e.g., Barth and Höller 1999; Barth 2002), but there is also increasing evidence from other arthropod groups that such filiform hairs play important roles in intraspecific communication (Heidelbach et al. 1991; Heidelbach and Dambach 1997; Santer and Hebets 2008). In order to explore this possibility for S. retrorsa, we conducted some supplementary analyses to demonstrate the plausibility of particle displacement functioning in S. retrorsa courtship (see Supplementary Material). Our preliminary calculations tentatively show that the leg waves of male $S$. retrorsa could induce air particle velocities of sufficient magnitude to be detected by the trichobothria on the metatarsus of a female at a separation of $\leq 65 \mathrm{~mm}$ (see Supplementary Figure 1). Furthermore, the observation that the number of courtship bouts is predictive of copulation success lends support to the hypothesis that the leg waving display is generating a nearfield signal that ultimately maintains signal efficacy across variation environmental condition. Nonetheless, although theoretically plausible, further studies are again necessary to confirm the use of near-field particle displacement in the multimodal courtship display of $S$. retrorsa.

The observed pattern of copulation success independent of signaling environment for $S$. retrorsa differs markedly from many previously examined Schizocosa, including S. uetzi, S. stridulans, and S. rovneri. All 3 of these species are more likely to copulate when environmental conditions permit the transmission of seismic signals (Hebets and Uetz 1999; Hebets 2005, 2008; Uetz et al. 2009). This apparent difference in seismic signal function and importance across species curiously coincides with both natural signaling substrates and the method of seismic signal production. To elaborate, S. uetzi and S. stridulans are both found on leaf litter and produce seismic signals mainly via stridulation (Stratton 2005). Schizocosa retrorsa, in contrast, is found mainly on pine litter, red clay, or sand and produces seismic signals via pedipalpal drumming. The seismic signaling substrate is also known to influence copulation success in $S$. retrorsa as pairs are more likely to copulate on their natural substrates of pine litter and red clay as compared with leaf litter (Hebets, Elias, et al. 2008). Although the emerging pattern suggests that the signaling environment has played a major role in shaping courtship form and function for at least some Schizocosa species, with currently available data, we are not yet able to fully elucidate the links between natural signaling substrate, seismic signal production, and seismic signal importance or function across Schizocosa species.

\section{Supplementary Material}

Supplementary Material appears following the References. It can also be accessed (by subscribers) at http://www.beheco. oxfordjournals.org/
Acknowledgments - This research was supported in part by funding from the National Science Foundation: IOS 0643179 to E. A. Hebets. We would like to thank P. Miller and G. Stratton for assistance in collecting spiders, and K. Swoboda for the care and maintenance of spiders in the laboratory. We would also like to thank K. Fowler-Finn, D. Wilgers, M. Adams, and S. Schwartz for comments on this manuscript; D. Eberl, J. A. C. Humphries, R. Hoy, and J. Bradbury for helpful and informative discussions regarding the near-field acoustics; and F. G. Barth for encouragement on the importance of near-field sound signaling in spiders.

\section{References}

Ayyagari LR, Tietjen WJ. 1986. Preliminary isolation of male-inhibitory pheromone of the spider Schizocosa ocreata (Araneae, Lycosidae). J Chem Ecol. 13:237-244.

Barth FG. 1982. Spiders and vibratory signals: sensory reception and behavioral significance. In: Witt PN, Rovner JS, eds., Spider communication: Mechanisms and ecological significance. Princeton: Princeton University Press. p. 67-122.

Barth FG. 2002. A spider's world: Senses and behavior. Berlin: Springer.

Barth FG, Höller A. 1999. Dynamics of arthropod filiform hairs. V. The response of spider trichobothria to natural stimuli. Philos Trans R Soc Lond B Biol Sci. 354:183-192.

Becker E, Riechert S, Singer F. 2005. Male induction of female quiescence/catalepsies during courtship in the spider, Agelenopsis aperta. Behaviour. 142:57-70.

Bennet-Clark HC. 1971. Acoustics of insect song. Nature. 234: 255-259.

Briers RA. 2003. MANTEL XLA. VBA add-in for Microsoft EXCEL, Version 1.2.4 [Internet]. Online at http://www.lifesciences.napier.ac.uk/staff/rob/software/index.html

Candolin U. 2003. The use of multiple cues in mate choice. Biol Rev. 78:575-595.

DeVoe RD. 1972. Dual sensitivities of cells in wolf spider eyes at ultraviolet and visible wavelengths of light. J Gen Physiol. 59:247-269.

DeVoe RD, Small RJW, Zvargulis JE. 1969. Spectral sensitivities of wolf spider eyes. J Gen Physiol. 54:1-32.

Dondale CD, Redner JH. 1978. Revision of the nearctic wolf spider genus Schizocosa (Araneida: lycosidae). Can Ent. 110:143-181.

Draud M, Macias-Ordonez R, Verga J, Itkowitz M. 2004. Female and male Texas cichlids (Herichthys cyanoguttatum) do not fight by the same rules. Behav Ecol. 15:102-108.

Elias D, Mason A, Hoy R. 2004. The effect of substrate on the efficacy of seismic courtship signal transmission in the jumping spider Habronattus dossenus (Araneae: Salticidae). J Exp Biol. 207:4105-4110.

Fleishman LJ. 1986. Motion detection in the presence and absence of background motion in an Anolis lizard. J Comp Physiol A. 159: 711-720.

Framenau V, Hebets E. 2007. A review of leg ornamentation in male wolf spiders, with the description of a new species from Australia, Artoria schizocoides (Araneae, Lycosidae). J Arach. 35:89-101.

Gaskett AC. 2007. Spider sex pheromones: Emission, reception, structures, and functions. Biol Rev. 82:27-48.

Gibson J, Uetz GW. 2008. Seismic communication and mate choice in wolf spiders: Components of male seismic signals and mating success. Anim Behav. 75:1253-1262.

Görner P, Andrews P. 1969. Trichobothrien, ein ferntastsinnesorgan bei webspinnen (Araneen). Z Vergl Physiol. 64:301-331.

Guilford T, Dawkins M. 1991. Receiver psychology and the evolution of animal signals. Anim Behav. 42:1-14.

Hebets E. 2005. Attention-altering signal interactions in the multimodal courtship display of the wolf spider Schizocosa uetzi. Behav Ecol. 16:75-82.

Hebets E, Papaj D. 2005. Complex signal function: Developing a framework of testable hypotheses. Behav Ecol Sociobiol. 57:197-214.

Hebets E, Uetz GW. 1999. Female responses to isolated signals 
from multimodal male courtship displays in the wolf spider genus Schizocosa (Araneae: Lycosidae). Anim Behav. 57:865-872.

Hebets E, Uetz GW. 2000. Leg ornamentation and the efficacy of courtship display in four species of wolf spider (Araneae: Lycosidae). Behav Ecol Sociobiol. 47:280-286.

Hebets EA. 2008. Seismic signal dominance in the multimodal courtship display of the wolf spider Schizocosa stridulans Stratton 1991. Behav Ecol. 19:1250-1257.

Hebets EA, Cuasay K, Rivlin PK. 2006. The role of visual ornamentation in female choice of a multimodal male courtship display. Ethology 112:1062-1070.

Hebets EA, Elias DO, Mason AC, Miller GL, Stratton GE. 2008. Substrate-dependent signaling success in the wolf spider, Schizocosa retrorsa. Anim Behav. 75:605-615.

Hebets EA, Stratton GE, Miller GL. 1996. Habitat and courtship behavior of the wolf spider Schizocosa retrorsa (Banks) (Araneae, Lycosidae). J Arach. 24:141-147.

Hebets EA, Wesson J, Shamble PS. 2008. Diet influences mate choice selectivity in adult female wolf spiders. Anim Behav. 76:355-363.

Heidelbach J, Dambach M. 1997. Wing-flick signals in the courtship of the African cave cricket Phaeophilacris spectrum. Ethology 103:827-843.

Heidelbach J, Dambach M, Böhm H. 1991. Processing wing flickgenerated air-vortex signals in the African cave cricket Phaeophilacris spectrum. Naturwissenschaften 78:277-278.

Johnstone RA. 1996. Multiple displays in animal communication: 'Backup signals' and 'multiple messages'. Philos Trans $R$ Soc Lond B Biol Sci.351:329-338.

Lindström M, Meyer-Rochow VB. 1987. Near infra-red sensitivity of the eye of the crustacean Mysis relicta. Biochem Biophys Res Commun. 147:747-752.

Møller AP, Pomiankowski A. 1993. Why have birds got multiple sexual ornaments. Behav Ecol Sociobiol. 32:167-176.

Owings DH, Morton ES. 1998. Animal vocal communication: A new approach. New York \& Cambridge: Cambridge University Press.

Partan S, Marler P. 1999. Communication goes multimodal. Science 283:1272-1273.

Partan S, Marler P. 2005. Issues in the classification of multimodal communication signals. Am Nat. 166:231-245.
Rowe C. 1999. Receiver psychology and the evolution of multicomponent signals. Anim Behav. 58:921-931.

Rowe C, Guilford T. 1999. Novelty effects in a multimodal warning system. Anim Behav. 57:341-346.

Rundus AS, Owings DH, Joshi SS, Chinn E, Giannini N. 2007. Ground squirrels use an infrared signal to deter rattlesnake predation. Proc Nat Acad Sci USA 104:14372-14376.

Santer RD, Hebets EA. 2008. Agonistic signals received by an arthropod filiform hair allude to the prevalence of near-field sound communication. Proc R Soc B Biol Sci. 275:363-368.

Scheffer S, Uetz GW, Stratton G. 1996. Sexual selection, male morphology, and the efficacy of courtship signalling in two wolf spiders (Araneae: Lycosidae). Behav Ecol Sociobiol. 38:17-23.

Shamble PS, Wilgers DJ, Swoboda KA, Hebets EA. 2009. Courtship effort is a better predictor of mating success than ornamentation for male wolf spiders. Behav Ecol. 20:1242-1251.

Shimozawa T, Murakami J, Kumagai T. 2003. Cricket wind receptors: Thermal noise for the highest sensitivity known. In: Barth FG, Humphrey JAC, Secomb TW, eds., Sensors and sensing in biology and engineering (Berlin: Springer), pp. 145-157.

Sokal RR, Rohlf FJ. 1995. Biometry: The principles and practice of statistics in biological research. 3rd ed. New York: W.H. Freeman and Company.

Stratton G. 2005. Evolution of ornamentation and courtship behavior in Schizocosa: Insights from a phylogeny based on morphology (Araneae, Lycosidae). J Arach. 33:347-376.

Uetz GW, Norton S. 2007. Preference for male traits in female wolf spiders varies with the choice of available males, female age and reproductive state. Behav Ecol Sociobiol. 61:631-641.

Uetz GW, Papke R, Kilinc B. 2002. Influence of feeding regime on body size, body condition and a male secondary sexual character in Schizocosa ocreata wolf spiders (Araneae, Lycosidae): Condition-dependence in a visual signaling trait. J Arach. 30:461-469.

Uetz GW, Roberts JA. 2002. Multisensory cues and multimodal communication in spiders: Insights from video/audio playback studies. Brain Behav Evol. 59:222-230.

Uetz GW, Roberts JA, Taylor PW. 2009. Multimodal communication and mate choice in wolf spiders: Female response to multimodal versus unimodal signals. Anim Behav. 78:299-305. 


\section{Supplementary Materials - 1}

\section{Estimating the detectability of near-field particle displace- ments induced by the courtship of male S. retrorsa.}

Our results suggest that the courtship display of male $S$. retrorsa wolf spiders can be perceived by females even when its seismic and visual components are ablated. In our manuscript we suggest that one possible explanation for this is that females detect this courtship in the near-field sound modality, and in this section we provide some estimations of the feasibility of this proposal. Male $S$. retrorsa courtship consists of a prominent leg wave, which could be a significant cause of near-field particle movements. To assess this hypothesis, we attempted to estimate: (1) what velocity of near-field particle displacement is generated by male leg-waving at characteristic distances seen during courtship, and (2) whether or not female tricobothria are capable of detecting particle displacements at the velocities generated by male leg-waving.

Leg waving by courting $S$. retrorsa males consists of the repeated lifting and lowering of typically one, but sometimes both, foreleg tips. In order to estimate air particle displacement induced by male $S$. retrorsa leg waves, we needed to quantify the kinematics of this behavior and the dimensions of the male foreleg. We recorded a typical bout of leg waving for one male, from the side at $500 \mathrm{fps}$ using a PCI 1000 digital high-speed video camera (RedLake Motionscope, San Deigo, CA, USA). This film was converted to AVI format and the position of the foreleg joints was tracked frame by frame as calibrated $x, y$ co-ordinates using ProAnalyst Lite software (Motion Engineering Company, Inc., Indianapolis, IN, USA). We quantified the dimensions of a male's foreleg by photographing a removed leg using a dissecting microscope and Spot Flex $64 \mathrm{MP}$ digital camera (Diagnostic Instruments, Inc., Sterling Heights, MI, USA). IMAGEPRO Discovery software was used to make measurements from the captured photographs (Media Cybernetics, Inc., Bethesda, MD, USA).

A single up and down wave had a mean period of approximately $73.8 \mathrm{~ms}$ (corresponding to $13.55 \mathrm{~Hz}$; $\mathrm{n}=10$ waves) and the foreleg tip moved with an amplitude of approximately $6 \mathrm{~mm}$ (although between consecutive waves the leg was held stationary and close to horizontal for $>100 \mathrm{~ms}$, meaning that leg waves repeated with an approximately $300 \mathrm{~ms}$ period $(\sim 3.33 \mathrm{~Hz}))$. In order to function as a communicative signal the air particles displaced by this leg movement must travel at a sufficiently fast velocity to adequately move the trichobothria of the receiving female. Using the above measurements, and the formulation used by Bennet-Clark (1971) to estimate particle velocities induced by the wing waves of courting male Drosophila, we treat the leg wave as an acoustic doublet source (Bennet-Clark 1971). Following Bennet-Clark's formulation, the acoustical power of such a source is given by

$$
I=(2 \pi f d)^{2} r_{m}
$$

where $I$ is the r.m.s. power expressed in $W ; f$ is frequency in $\mathrm{Hz}$; $d$ is the displacement in $\mathrm{m}$; and $r_{m}$ is the acoustic resistance of the source in $\mathrm{kg} \mathrm{s}^{-1}$. For S. retrorsa, $f$ is $13.55 \mathrm{~Hz}, d$ is $0.006 \mathrm{~m}$, and $r_{m}$ is the product of the area of one side of the leg $(8.78 \mathrm{x}$ $\left.10^{-6} \mathrm{~m}^{2}\right)$, and the specific resistance of a source whose radius is $3 \times 10^{-4}$ times the wavelength of the sound $(25.11 \mathrm{~m})$; (a resistance of $2 \times 10^{-5} \mathrm{~kg} \mathrm{~m}^{-2} \mathrm{~s}^{-1}$ read from Bennet-Clark (1971)'s figure 1). These variables yield an acoustic power of $4.58 \times 10^{-11}$ W (Bennet-Clark 1971). In cases where the inverse square law applies, where acoustic power is inversely proportional to the square of the distance to the source (when $r>1 / 3 \lambda, r=$ distance from the source and $\lambda=$ wavelength), acoustical power can be expressed as particle velocity by

$$
I=v^{2} \rho c
$$

where $v$ is velocity, $\rho$ is the density of air $\left(1.229 \mathrm{~kg} / \mathrm{m}^{3}\right)$, and $c$ is the speed of sound $(340.29 \mathrm{~m} / \mathrm{s})$. However when $\mathrm{r}<1 / 6 \lambda$ as is the case with the leg waving of $S$. retrorsa, particle velocity must be increased by a factor of

$$
\left(\frac{\lambda}{2 \pi r}\right)^{2}
$$

yielding a velocity of $2984.28 \mathrm{~mm} / \mathrm{s}$ at a distance of $10 \mathrm{~mm}$ dropping to $8.70 \mathrm{~mm} / \mathrm{s}$ at $70 \mathrm{~mm}$ from the source (Figure ESM1).

The trichobothria of $S$. retrorsa have not been studied, so to estimate whether the above particle velocities would be theoretically detectable we use data from the much larger Ctenid spider, Cupiennius salei. In this species, both long $(>400 \mu \mathrm{m})$ and short $(<400 \mu \mathrm{m})$ trichobothria (of group D1 on the dorsal metatarsus of the walking leg) require a deflection of only $0.4-0.5^{\circ}$ at $13.55 \mathrm{~Hz}$ to elicit a response in the underlying mechanoreceptors (Barth and Holler 1999). How easily these trichobothria are deflected by varying air particle velocities depends to a large extent on their length: an $850 \mu \mathrm{m}$ trichobothrium (also of metatarsal group MeD1) requires an approximately 10-15 $\mathrm{mm} / \mathrm{s}$ particle velocity to deflect it $2.5^{\circ}$, a $500 \mu \mathrm{m}$ trichobothrium of the same group requires an approximately $40-50 \mathrm{~mm} / \mathrm{s}$ particle velocity to deflect it the same amount (Barth et al. 1993). How easily the hairs of Cupiennius are deflected also depends on their position and arrangement. However, here we use $10-15 \mathrm{~mm} / \mathrm{s}$ as a theoretical threshold detectable particle velocity for comparison with our calculations above. Using this threshold, S. retrorsa leg waves could, theoretically, be detected by the trichobothria on the metatarsus of a female at a separation of $\leq 65 \mathrm{~mm}$. The length of the trichobothria is $S$. retrorsa will determine their exact sensitivity, which may be less than that extrapolated from C. salei, however, we have been conservative in using a much larger threshold angular deflection than would be at the limit of detectability.

\section{References}

Barth FG, Wastl U, Humphrey JAC, Devarakonda R. 1993. Dynamics of arthropod filiform hairs. II. Mechanical properties of spider trichobothria (Cupiennius salei Keys.). Philos Trans $R$ Soc Lond., B 340: 445-461.

Barth FG, Höller A. 1999. Dynamics of arthropod filiform hairs. V. The response of spider trichobothria to natural stimuli. Philos Trans R Soc Lond., B. 354: 183-192.

Bennet-Clark HC. 1971. Acoustics of insect song. Nature 234: 255-259. 


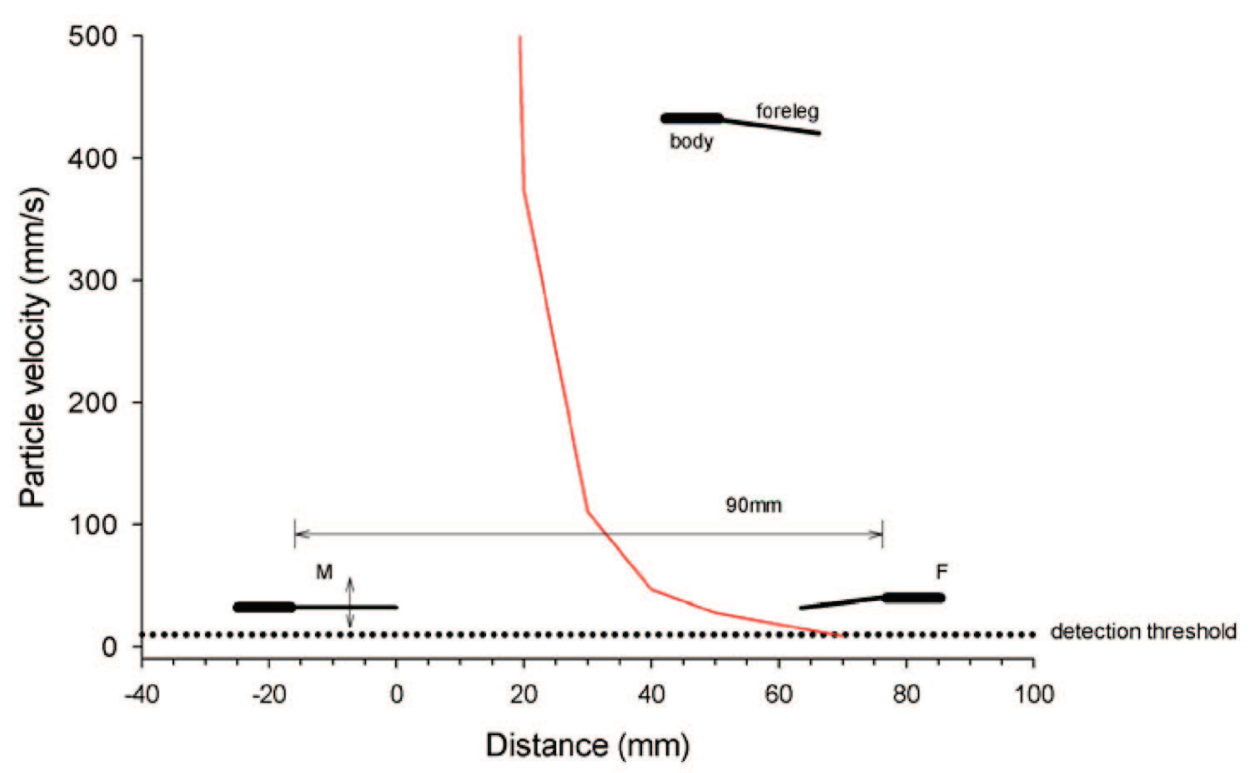

Supplementary Figure 1: Calculated estimates of the velocity of air particles displaced by the leg waving behavior of male S. retrorsa at varying distance from the source. The dotted line represents the detection threshold $(10 \mathrm{~mm} / \mathrm{s})$ from measurements of $C$. salei. Particle velocities are above trichobothria threshold detection levels at distances less than $65 \mathrm{~mm}$ from the source. If the source is assumed to be the tip of the male's waving leg, and the receiver the trichobothria on the metatarsus of the female's foreleg, this maximum distance corresponds to an approximate spacing of $\sim 90 \mathrm{~mm}$ between the body of the signaler and the body of the signal perceiver $(65 \mathrm{~mm}$ separation between leg tip of waving male, and metatarsal trichobothria of receiving female). 